\title{
The association between leafy vegetable consumption and incidence of metabolic syndrome and its symptoms: a systematic review of randomised controlled and observational trials
}

\author{
E.N. Muriuki ${ }^{1}$, B. Celik ${ }^{1}$, G.G.C. Kuhnle ${ }^{1}$ and C.E. Mills ${ }^{1}$ \\ ${ }^{1}$ Hugh Sinclair Unit of Human Nutrition, University of Reading, Whiteknights, Reading, UK
}

Metabolic syndrome (MetS) is a cluster of cardiovascular risk factors which include dyslipidemia, hypertension, hyperglycemia, and obesity, its presence is linked to an increase in cardiovascular disease and Type-2 diabetes ${ }^{(1)}$. Leafy vegetables such as spinach, rocket and Swiss chard have been shown to reduce symptoms of metabolic syndrome ${ }^{(2)}$, possibly because they contain bioactive compounds such as nitrates, dietary fibre and flavonoids. Here, we performed a systematic review to investigate if the consumption of leafy vegetables prevents the incidence of MetS or its symptoms.

The Web of Science, Scopus, and PubMed were searched from inception to September 2020. The inclusion criteria was randomised control trials (RCT) and prospective cohort studies, testing the consumption of leafy vegetables versus a control in healthy male or female adults on MetS or any of its symptoms; only papers written in English were included. The search terms included names of different leafy vegetables and terms describing MetS and its symptoms. The risk of bias was assessed using the Cochrane tool for the RCTs and the Newcastle-Ottawa Scale was used for the cohort studies

From the database searching, 3,684 records were retrieved, 459 duplicates were removed. The titles and abstracts of 3,225 articles were screened and 3,072 articles were removed. Full text screening was performed for 153 articles. Of these, 148 articles were excluded, the main reasons for exclusion were, studies with participants with chronic illness $(n=63)$ and studies involving plant extracts $(n=27)$. Finally, 4 RCTs ( 3 acute trials and 1 chronic) and 1 cohort study remained. An inverse association between the consumption of leafy vegetables and the incidence of hypertension was observed, odds ratio $0.63(95 \%$ CI $0.41,0.98)$ in the cohort study ( $\mathrm{n}=1544)$. A reduction in systolic blood pressure was observed after the consumption of leafy vegetables in one acute RCT ( $\mathrm{n}=26$ ). No effect of consumption of vegetables on blood glucose was observed in one acute RCT $(n=10)$ while in another acute $(n=12)$ and the chronic $(\mathrm{n}=90)$ trial, a reduction in postprandial and fasting blood glucose was observed respectively. There were no trials assessing, obesity, waist circumference, or MetS as a whole. The studies were heterogeneous since they involved different types of vegetables given at different doses and at different durations. The risk of bias assessment shown some concerns in the RCT trials since there was no blinding.

Conclusions about the impact of consumption of green leafy vegetables on MetS and its symptoms cannot be drawn from this review due to the limited number of studies and high level of heterogeneity of included papers. There is need to carry out more RCTs and observational trials on the impact of consumption of leafy vegetables as a single food component on MetS and its symptoms.

\section{References}

1. O'Neill S \& O'Driscoll L (2015) Obes Rev 16, 1-12.

2. Hosseinpour-Niazi S, Bakhshi B, Betru E, et al. (2019) World J Diabetes 10 (6), 362-375. 